\title{
La gestión de la reputación online de las marcas hospitalarias: una propuesta de modelo
}

\author{
Ospitale-marken online ospearen \\ kudeaketa:eredu-proposamen bat
}

\section{The online reputation management of hospital brands: a model proposal}

\section{Pablo Medina Aguerrebere ${ }^{1}$}

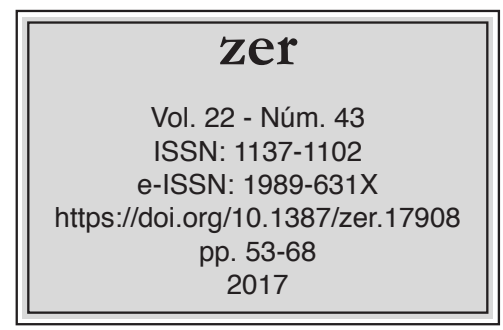

Recibido el 6 de junio de 2017, aceptado el 28 de septiembre de 2017.

Resumen: El objetivo de este artículo de revisión bibliográfica es analizar el impacto de las redes sociales en la creación de la reputación online de una marca hospitalaria. El artículo propone el modelo PMA de gestión de la reputación online para marcas hospitalarias, y concluye afirmando que la existencia de un equipo multidisciplinar, la proyección de la marca en el largo plazo y la implicación de los profesionales de la salud son aspectos determinantes para que el Director de Comunicación de un hospital pueda crear una marca reputada.

Palabras clave: Salud, comunicación corporativa, marca, reputación, redes sociales.

\section{Laburpena}

Berrikuspen bibliografikoan oinarritutako artikulu honen helburua da sare sozialek ospitalemarka baten online ospearen eraketan duten inpaktua aztertzea. Artikuluak PMA kudeaketaeredua proposatzen du ospitale-marken online ospea kudeatzeko, eta ateratzen duen ondorioa da ospitale bateko komunikazio-zuzendariak alderdi erabakigarri hauek erabil ditzakeela ospe oneko marka bat sortzeko: diziplinarteko talde bat izatea, markaren epe luzerako proiekzioa, eta osasunaren arloko profesionalen inplikazioa.

Gako-hitzak: Osasuna, komunikazio korporatiboa, marka, erreputazioa, sare sozialak.

\begin{abstract}
The objective of this bibliographic review paper is to analyse the impact of social media in the creation of the online reputation of a hospital brand. This paper proposes the PMA model
\end{abstract}

$\overline{1}$ Universidad de Ginebra (Suiza), pablo.medina@unige.ch 
for online reputation management in hospital brands and concludes that the existence of a multidisciplinary team, the brand projection in the long term and the involvement of health professionals are determining factors, which help the Hospital Head of Communication to create a reputed brand.

Keywords: Health, corporate communication, brand, reputation, social media. 


\section{Introducción}

En la mayor parte de los países, el contexto hospitalario ha cambiado radicalmente durante estos últimos años: déficit de algunos hospitales públicos, creación de grupos hospitalarios con presencia internacional, desarrollo de los hospitales privados, exigencias crecientes de los pacientes, etc. Este nuevo paradigma empresarial obliga a los hospitales a invertir en comunicación institucional para crear marcas reputadas, sólidas y creíbles que les ayuden a reforzar sus posicionamientos estratégicos en sus mercados de referencia. En este contexto, varias organizaciones hospitalarias recurren a las redes sociales para acelerar el proceso de construcción de una marca reputada. El objetivo de este artículo es comprender el verdadero impacto que tienen las redes sociales en la construcción de la reputación online de una marca hospitalaria. La hipótesis principal de esta investigación es que las redes sociales se han convertido en la herramienta prioritaria para construir la reputación corporativa del hospital. Con el objetivo de verificar si dicha hipótesis es cierta, se ha llevado a cabo una investigación cualitativa basada en una metodología de revisión bibliográfica actualizada sobre los principales trabajos publicados por los autores expertos en el ámbito de la comunicación institucional, la marca, las redes sociales y la comunicación de salud. El artículo se divide en dos partes: en primer lugar, se realiza una revisión bibliográfica sobre los conceptos de marca hospitalaria, arquitectura de marca, reputación y redes sociales; y, en segundo lugar, se propone un modelo de gestión de la reputación online de la marca hospitalaria.

\section{La marca hospitalaria}

En el contexto hospitalario actual, caracterizado por el cambio constante en las expectativas de los pacientes y por la consolidación de los empleados del hospital como la fuerza comunicativa más importante de la organización, resulta necesario que la marca corporativa del hospital se convierta en un activo intangible capaz de unir y liderar a todos los empleados de la organización (Naveen, Anil y Smruthi, 2014). La gestión estratégica de la marca se ha convertido en una prioridad para los hospitales, de ahí que la mayoría de ellos apuesten por definir y divulgar su arquitectura de marca a todos sus stakeholders -empleados, pacientes, autoridades públicas, etc.- (Medina, 2015). Dicha arquitectura de marca se compone de cinco elementos principales: identidad, valores, misión, visión y cultura (Nieto, 2005). La identidad es una fuente de ventaja corporativa ya que indica la esencia de una organización (He y Balmer, 2008) y está íntimamente relacionada con otros elementos corporativos como la imagen de la empresa y la visión (Van Riel y Fombrun, 2007), así como con la estrategia de la propia organización: entre ambas existe una relación simbiótica, reciproca y dinámica (He y Balmer, 2013). Los valores de una organización son aquellas ideas que son compartidas por todos los empleados y que les ayudan a tener éxito en sus proyectos individuales y/o colectivos (Andersen, 2010). Los valores siempre están presentes en la organización y se plasman en todos y cada uno de los comportamientos y procesos de trabajo que en esta se llevan a cabo, expresando de este modo la forma de ser y pensar única que tiene la organización (Jaakson, 2010). La misión indica los objetivos que persigue una organización y se nutre de conceptos estratégicos que 
permiten explicar a los stakeholders cómo se aplica dicha misión en la práctica (Cady, Wheeler, De Wolf y Brodke, 2011). La definición de la misión supone varios beneficios para la organización: aporta sentido a los objetivos perseguidos por la organización, ayuda a centrar los recursos en lo realmente prioritario, mejora la comunicación con los stakeholders internos y externos, y ayuda a describir los valores que guían a los empleados (Desmidt, Prinzie y Decramer, 2011). La visión define el camino que debe seguir la organización para alcanzar sus objetivos de largo plazo, los cuales van más allá de los establecidos en la misión (Singal, Jain, 2013). La visión no solo debe ser breve, sino que además debe describir un objetivo principal que abarque a todos los intereses de la organización, que motive a sus empleados y que proyecte a dicha organización en el largo plazo (Kantabutra y Avery, 2010). Por último, la cultura se puede definir como una narración realizada por los líderes de la organización que se convierte en parte de las creencias de todos los empleados, así como en fuente de motivación (Marshall y Adamic, 2010). En el entorno hospitalario, la cultura corporativa está determinada por la capacidad del hospital para alinear la misión, los valores y las prácticas profesionales de todos los días; por eso, solo los hospitales que implantan una cultura coherente consiguen tener éxito en sus actividades, es decir, ofrecer un servicio de salud de alta calidad y con valor añadido (Nelson, Taylor y Walsh, 2014).

Los hospitales definen la arquitectura de marca con el objetivo de difundir ante los stakeholders internos y externos una imagen de marca univoca y coherente de la organización. Las relaciones de una empresa con sus stakeholders generan un conocimiento intangible denominado "capital relacional" que puede aportar mucho valor a la organización (Tejedo, 2013), pero también generan percepciones que determinan la opinión que dichos stakeholders tienen sobre la organización. Según Van Riel (1998), las impresiones personales, la comunicación interpersonal y la comunicación masiva se combinan para producir una mezcla de impresiones reales y paralelas cuya totalidad forman la imagen de marca. Dicha imagen está considerada como la identidad percibida por parte de los stakeholders (He y Balmer, 2008). Para un centro hospitalario, los dos stakeholders más importantes son los empleados y los pacientes. Las acciones de comunicación de marca destinadas a los empleados son vitales para la organización ya que le ayudan a generar reconocimiento, aumentan la cultura positiva y el compromiso de los empleados, mejoran la gestión del cambio, e influyen en la atracción y retención de los mejores empleados (Drake, Gulman y Roberts, 2005). Los empleados son un target clave a la hora de gestionar la imagen de marca de una organización ya que representan, de modo tangible, los conceptos intangibles de misión, visión, valores y cultura (Abratt y Kleyn, 2012). Por su parte, los pacientes son cada vez más selectivos a la hora de elegir sus productos de salud, así como los centros hospitalarios a los que acudir (Sparer, 2011). Cuando acuden a un hospital, los pacientes viven experiencias de servicio personales e íntimas, de ahí la importancia de que el hospital conecte emocionalmente con ellos a través de la comunicación de marca ya que dicha conexión puede mejorar el compromiso afectivo de los pacientes con la organización hospitalaria (Kemp, Jilipalli y Becerra, 2014). Las acciones de comunicación de marca destinadas a conectar emocionalmente con el paciente son fundamentales para cualquier hospital, ya que le permiten influenciar directamente en los procesos de toma de decisión que este lleva a cabo, así como en el boca a oreja generado sobre el hospital (Naveen, Anil y Smruthi, 2014). 
La gestión estratégica de la marca aporta varios beneficios al hospital: entre todos ellos, destaca la reputación de la organización ante los distintos stakeholders, especialmente ante los pacientes y los empleados. La reputación consiste en una verdadera evaluación que los stakeholders realizan sobre la organización, sus productos y servicios (Van Riel y Fombrun, 2007). En el entorno hospitalario, la buena reputación se construye a partir de la confianza que los pacientes manifiestan hacia la organización y sus tratamientos, así como a partir del conocimiento que dichos pacientes tienen sobre la propia organización (Hoon Kim, Sik Kim, Yul Kim, Ho Kim y Hou Kang, 2008). La reputación influye positivamente en la competitividad de una organización, la cual puede ser definida como la fortaleza de una organización para tener éxito en su mercado de referencia (O’Cass y Weerawardena, 2010). Las organizaciones hospitalarias que gestionan eficazmente sus acciones de comunicación de marca consiguen desarrollar la reputación corporativa, la competitividad de la organización, así como la credibilidad de esta última ante sus distintos grupos de interés. Y dicha credibilidad desempeña un papel clave en la gestión de la gobernanza corporativa de la organización (Trong, 2014).

\section{El rol de las redes sociales como agentes dinamizadores de la marca hospitalaria}

La comunicación institucional en el entorno hospitalario siempre se ha caracterizado por el uso de formatos clásicos (revistas internas, tablón de anuncios, newsletter, página web, etc.) y por el recurso a enfoques periodísticos centrados exclusivamente en la actualidad del hospital y con un marcado carácter unidireccional. Este enfoque, más propio del periodismo que de la comunicación institucional, no responde a las nuevas necesidades estratégicas que tienen estas organizaciones, como por ejemplo la gestión de la marca, el desarrollo de la reputación, o la gestión de las relaciones con los stakeholders. El paciente se ha convertido en un verdadero líder de opinión capaz de influenciar directamente en la imagen pública de los hospitales (Becerra, Reina, Victoria, 2015), de ahí la importancia de que estas organizaciones adopten un enfoque más estratégico a la hora de gestionar la comunicación institucional. En este contexto, la aparición de las redes sociales ha supuesto un verdadero impacto para los profesionales de la comunicación institucional que trabajan en los entornos hospitalarios. Estas herramientas constituyen una oportunidad única para ayudar a los hospitales a superar el enfoque periodístico e implantar una comunicación institucional que gestione la marca y la reputación del hospital de un modo más dinámico. Cinco realidades permiten realizar esta afirmación: 1) el nuevo rol comunicativo del profesional de la salud, 2) la mayor implicación del paciente, 3) el desarrollo de la educación en salud, 4) la mejora en la investigación de mercados y 5) la versatilidad de formatos.

El profesional de la salud. Las redes sociales tienen un impacto directo en la actividad clínica del profesional de la salud, ya que mejoran la toma de decisiones conjuntas que él realiza con el paciente y porque además influyen positivamente en la calidad del servicio médico ofrecido a este último (Lim, 2016). Además, son muy útiles a la hora de solucionar ciertos problemas de salud ya que permiten difundir rápidamente información médica a varias personas y porque mejoran la comunicación 
médico-paciente (Matarín Jiménez, 2015). Las redes sociales han cambiado radicalmente las relaciones comunicativas que el profesional de la salud establece con el paciente. Dichos profesionales se convierten en los principales comunicadores del hospital, es decir, en verdaderos embajadores de la marca hospitalaria capaces de dinamizar la comunicación institucional del hospital. Los profesionales de la salud usan las redes sociales para interactuar con el paciente y así mejorar el tratamiento médico, enviarle información médica de calidad y motivarle para seguir el tratamiento establecido (George, Rovniav y Kraschnewski, 2013). Los profesionales de la salud apuestan por la comunicación a través de las redes sociales porque los pacientes exigen mayor rapidez y calidad (Britnell, 2011), pero también porque gracias a esta herramienta, pueden fomentar su reputación personal como expertos en salud. Asi, según Alpert y Womble (2016), los profesionales de la salud, gracias a las redes sociales, comparten información sobre nuevas investigaciones, contactan con otros compañeros del sector y desarrollan la relación con el paciente en un nivel que va más allá de la mera consulta médica en el hospital.

El paciente. Los pacientes suelen utilizar Twitter como medio para conocer mejor un tema médico que les preocupa, así como para intercambiar opiniones; en cambio, Facebook lo suelen usar fundamentalmente para encontrar un apoyo social (Antheunis, Tates y Nieboer, 2013). Los pacientes comparten en las redes sociales información médica muy especializada sobre temas tan variados como los síntomas de una patología, la medicación, los protocolos del tratamiento y los procedimientos médicos (Gage-Bouchard, LaValley, Mollica y Beaupin, 2016). Gracias a las redes sociales, los pacientes han roto la temporalidad en sus relaciones comunicativas con los profesionales de la salud que trabajan en un centro hospitalario. Así, por ejemplo, en el caso de Twitter, una vez que los hospitales establecen relación con los ciudadanos a través del perfil corporativo de la organización en dicha red social, estos últimos suelen mostrar un gran interés hacia la información relativa a la actividad del hospital, sus eventos y la actividad de los profesionales de la salud (Rando Cueto, Paniagua Rojano, De las Heras Pedrosa, 2016). Las redes sociales han transformado radicalmente el tipo de comunicación que el hospital establece con sus pacientes (Ratzan, 2011); ahora son los pacientes los que asumen la iniciativa en las relaciones comunicativas establecidas con el hospital, lo cual exige a estas organizaciones repensar sus estrategias de comunicación de marca para adaptarlas a este nuevo dinamismo comunicativo (Kietzmann, Hermkens, McCarthy y Silvestre, 2011).

La educación en salud. El desarrollo de Internet ha permitido que los pacientes tengan espacios propios para explicar sus vivencias médicas de un modo completamente interactivo (Visa Barbosa y Coll-Planas, 2015). En el ámbito hospitalario, una de las principales funciones de las redes sociales es la difusión de información médica que permite al paciente formarse en temas de salud para así adoptar comportamientos más responsables con el cuidado de su propia salud (Vance, 2009). Si bien es cierto que no toda la información difundida en dichas redes es precisa ni está contrastada (Pirraglia y Kravitz, 2012), se puede afirmar que las redes sociales facilitan enormemente la educación del paciente en temas médicos, lo cual mejora a su vez la relación comunicativa que éste mantiene con el profesional de la salud cuando acude al hospital ya que dicha relación es más simétrica (Smailhodzic, Hooijsma, Boonstra y Langley, 2016). La mejor formación del paciente en temas sanitarios 
permite aumentar su compromiso con el profesional de la salud a la hora de participar en el proceso de toma de decisiones sobre el tratamiento médico, el seguimiento del mismo y su posterior evaluación (Fordis, Street, Volk y Smith, 2011), lo cual contribuye a reforzar los lazos comunicativos entre el paciente, el profesional de la salud y el centro hospitalario.

Investigación de mercados. Las redes sociales ponen en evidencia el enorme conocimiento sobre temas médicos que reside en las colectividades de pacientes (Metzger y Falnagin, 2011). Gracias a las redes sociales, los hospitales pueden conocer mejor a sus pacientes, no solo desde un punto de vista médico, sino también comportamental. Según Bernhardt, Mays y Kreuter (2011), las redes sociales han creado la generación de e-pacientes más implicada con su salud de toda la historia, lo que les lleva a difundir constantemente información sobre temas médicos, preocupaciones, percepciones, etc. Por tanto, las redes sociales se han convertido en una fuente de información muy valiosa para los hospitales que desean conocer mejor a sus pacientes y adaptar la comunicación institucional a sus necesidades y exigencias. Estas herramientas permiten al hospital comprender el porqué de las decisiones que toma el paciente, pero también el porqué de la imagen que éste tiene sobre el hospital: así, una de las tendencias en redes sociales de hospitales consiste en solicitar a los pacientes que evalúen el servicio médico recibido, el trato ofrecido por los profesionales de la salud, su nivel de satisfacción con el hospital, y que expliquen el porqué de cada una de las evaluaciones (Gao, McCullough, Agarwal y Jha, 2012).

Versatilidad de formatos. La simplicidad de uso y consulta de la información en las redes sociales supone un valor añadido para los pacientes que desean simplificar sus procesos de búsqueda de información médica (Rothberg, Morsi, Benjamin, Pekow y Lindenauer, 2008). Además, las redes sociales, al presentar la información en formatos distintos al texto, permiten difundir información médica a audiencias con necesidades especiales, como es por ejemplo el caso de los vídeos, los cuales son muy útiles para divulgar conocimientos médicos a pacientes con escasa formación (Adams, 2010). Esta versatilidad de formatos supone una oportunidad estratégica para todos aquellos hospitales que desean divulgar información científica de un modo más ameno y dinámico. La pluralidad de formatos y la multitud de aplicaciones que ofrecen las redes sociales las han convertido en herramientas capaces de solucionar problemas complejos de salud pública (Salathé, Freifeld, Mekaru, Tomasulo y Brownstein, 2013).

\section{Modelo de gestión reputacional de la marca hospitalaria 2.0}

El Director de Comunicación de un hospital asume como responsabilidad principal la gestión efectiva de la reputación corporativa de la marca hospitalaria. Según Chun (2005), la reputación se compone de tres elementos principales -imagen, identidad transmitida e identidad deseada-, los cuales, a menudo, no son coherentes entre sí, lo que dificulta que los stakeholders de la organización tengan una imagen unívoca sobre ésta. La reputación corporativa es un concepto intangible que las organizaciones intentan evaluar y medir con indicadores cuantificables (Van Riel y Fombrun, 2007) ya que se trata de un elemento estratégico capaz de determinar el tipo de relaciones institucionales que la organización establece con sus diferentes stakeholders (Fombrun, Gardberg y Sever, 2000). 
En el contexto hospitalario, el uso masivo de las redes sociales por parte de los stakeholders, especialmente los pacientes, obliga a las organizaciones hospitalarias a reinventar sus modelos de gestión de la reputación de marca. Según Dijkmans, Kerhof y Beukeboom (2014), el uso de las redes sociales por parte de los stakeholders de la organización influye directamente en el compromiso que éstos pueden establecer con la organización, así como en la reputación online de la misma. Gracias a las redes sociales, los stakeholders de una organización pueden opinar libremente sobre las acciones que ésta realiza, sin necesidad de pasar por los intermediarios habituales, como eran los medios de comunicación (Cormode y Krishnamurthy, 2008). Esta nueva realidad obliga a los hospitales a rediseñar sus estrategias de comunicación institucional para así salvaguardar y potenciar la reputación online de sus marcas. La gestión de la reputación online de una marca implica varias acciones, como por ejemplo interactuar con varias personas en las redes sociales, crear contenido para compartir en dichas redes, monitorear lo que dicen los stakeholders, analizar sus diálogos, gestionar los contenidos negativos que difunden sobre la institución y actualizar y desarrollar las ideas más interesantes que los stakeholders comentan sobre la organización (Dijkmans, Kerhof y Beukeboom, 2014). La gestión de la reputación online de la marca hospitalaria se ha convertido en una prioridad máxima para el Director de Comunicación de cualquier hospital; sin embargo, él no es la única persona implicada en dicha labor, ya que la misma concierne a todos los empleados de la organización.

Con el objetivo de responder eficazmente a las nuevas necesidades comunicativas que tienen los hospitales, se propone el Modelo PMA para gestionar la reputación online de las marcas hospitalarias. Este modelo se compone de nueve fases diferentes y complementarias que deben ser lideradas por el Departamento de Comunicación del centro hospitalario, en estrecha colaboración con los órganos directivos del mismo, así como con los departamentos médicos y de administración (ver Imagen 1. Modelo PMA de reputación online en hospitales).

Imagen 1. Modelo PMA de reputación online en hospitales.

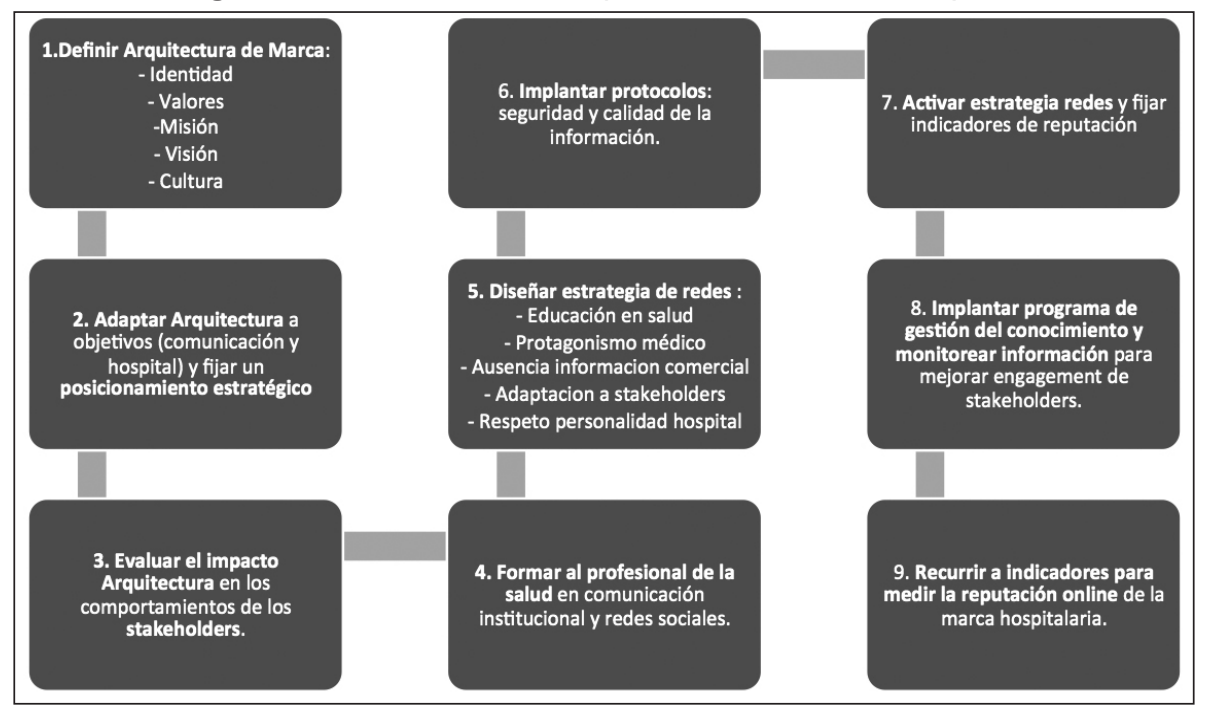

Fuente: Elaboración propia. 
Fase 1. Definición de la arquitectura de marca. El Director de Comunicación del hospital, en colaboración con los directivos del mismo y los responsables de los diferentes departamentos -médicos y de administración-, debe liderar un proceso colectivo que permita definir la arquitectura de marca del hospital, es decir, la identidad, los valores, la misión, la visión y la cultura. La identidad de una organización debe estar alineada con la misión, la visión y la estrategia de la organización (Ditlevesen, 2012), pero también con su cultura, la cual forma parte del negocio de la empresa ya que impulsa a los empleados a realizar bien su trabajo (Whiteley, Price y Palmer, 2013). Definir la arquitectura de marca y garantizar la coherencia entre todos los elementos que la integran constituye un primer paso fundamental para la correcta gestión de la reputación online del hospital.

Fase 2. Adaptación de la arquitectura de marca. Una vez definida dicha arquitectura, el Director de Comunicación debe analizar los objetivos globales perseguidos por el hospital y por sus departamentos estratégicos, así como los perseguidos por el propio Departamento de Comunicación, para armonizar y adaptar la arquitectura de marca a las metas colectivas perseguidas por la organización hospitalaria. El resultado de este proceso es la fijación de un posicionamiento estratégico que garantice la supervivencia del hospital en el largo plazo. Las empresas competitivas son capaces de entender su negocio y volcar dicho conocimiento en el desarrollo de una estrategia coherente con su cultura corporativa (Klein, 2011).

Fase 3. Evaluación del impacto de la arquitectura de marca en los stakeholders. El Director de Comunicación debe plasmar en un documento oficial la definición de todos los elementos integrantes de la arquitectura de marca. Y, posteriormente, debe organizar sesiones de trabajo con los distintos stakeholders del hospital (empleados, pacientes, autoridades públicas, etc.) para explicarles qué implicaciones practicas tiene dicha arquitectura en el día a día de todos y cada uno de ellos. Este proceso es clave para que el hospital pueda construir la marca de un modo colectivo junto a sus grupos de interés. La imagen de marca comprende el conocimiento del consumidor, y de otros stakeholders, sobre los productos y atributos intangibles de la marca (Lee, Lee y Wu, 2011), de ahí el interés en que los hospitales usen un enfoque más estratégico a la hora de gestionar la exposición social de su marca (Park, Rodgers y Stemmle, 2013).

Fase 4. Formación del profesional de la salud en comunicación institucional. El Departamento de Comunicación del hospital debe organizar sesiones de formación en comunicación institucional y gestión estratégica de redes sociales dirigidas a los profesionales de la salud que trabajan en el centro hospitalario. Estos profesionales son los comunicadores más activos del hospital (consultas presenciales, consultas online, entrevistas en medios de comunicación, etc.), de ahí el interés en que puedan ejercer con garantías su rol de embajador de marca del hospital. Las organizaciones sanitarias deben apostar por la formación de sus empleados en áreas de comunicación, como por ejemplo las redes sociales y la gestión de la reputación (Würz, Nurm y Ekdahl, 2013), para así comprender el verdadero potencial de estas herramientas en el establecimiento de relaciones de confianza con los stakeholders (Griffis, Kilaru, Werner, Asch, Hershey, Hill, Ha, Sellers, Mahoney y Merchant, 2014).

Fase 5. Diseño de la estrategia de redes. La comunicación online sobre temas médicos tiene un gran impacto en las actitudes y comportamientos de los usuarios (Peñafiel, Pastor, Camacho, 2014). Para asegurar el impacto de la comunicación en 
redes sociales, el Director de Comunicación debe elaborar una estrategia de redes sociales que permita al hospital alcanzar sus objetivos de comunicación institucional previamente definidos en el Plan de Comunicación. Para ello, dicha estrategia debe basarse en cinco pilares fundamentales: difundir información que permita al paciente mejorar su educación en salud; otorgar el protagonismo comunicativo a los profesionales de la salud, permaneciendo los integrantes del departamento de comunicación en un segundo plano; difundir únicamente información de interés para los stakeholders y sin ningún carácter comercial; adaptar el tono y los mensajes a los distintos stakeholders del hospital; y respetar la personalidad del hospital, es decir, su arquitectura de marca. Los contenidos de salud difundidos en redes sociales deben basarse en un enfoque comprensivo y de evidencia (Gibbons, Fleisher, Slamon, Bass, Kandadai y Beck, 2011), el cual es clave para construir una marca reputada.

Fase 6. Implantación de protocolos. La comunicación del hospital en las redes sociales debe basarse en protocolos previamente definidos que garanticen, por un lado, la seguridad y la calidad de la información difundida; y, por otro, la coherencia de dicha información con los objetivos de comunicación y organizacionales perseguidos por el hospital. Las organizaciones sanitarias deben profesionalizar los contenidos difundidos en redes sociales para así satisfacer las necesidades informativas de los distintos stakeholders (McCarroll, Armbruster, Chung, Kim, McKenzie y Von Gruenigen, 2014). Y para ello, resulta fundamental instaurar políticas y guías de uso de las redes sociales que ayuden a todos los empleados a comunicarse de un modo efectivo con los distintos grupos de interés de la organización (Campbell y Craig, 2014).

Fase 7. Lanzamiento de la campaña de redes. Una vez que el Director de Comunicación ha realizado con éxito las seis fases previas, el siguiente paso consiste en fijar los indicadores para medir la reputación (imagen percibida por los stakeholders, capacidad para atraer empleados, aumento de la cuota de mercado, etc.) y, posteriormente, activar la campaña de comunicación en redes sociales. Los hospitales no solo deben apostar por medir la reputación, sino también por medir el impacto que tiene la comunicación en redes sociales en la calidad del servicio médico ofrecido al paciente (Greaves, Ramirez, Millett, Darzi y Donaldson, 2013).

Fase 8. Implantación de un programa de gestión del conocimiento. Las acciones de comunicación en redes sociales generan una gran cantidad de información (percepciones de los stakeholders, quejas y críticas, oportunidades de negocio, etc.) que debe ser procesada y gestionada correctamente para, posteriormente, utilizarla en las futuras acciones de comunicación y así mejorar el engagement de los stakeholders con el hospital. Monitorear correctamente la información difundida en redes ayuda al hospital a orientar correctamente sus acciones estratégicas para mejorar la reputación online de la marca. Además, los hospitales deben gestionar dicha información para ponerla al servicio del profesional de la salud y ayudarle asi a mejorar su trabajo $\mathrm{y}$, por tanto, el servicio ofrecido al paciente (Bubien, 2015).

Fase 9. Medición de la reputación online. El Departamento de Comunicación del hospital debe recurrir a los indicadores de reputación previamente fijados para medir este aspecto intangible, no solo después de la ejecución de la campaña, sino también durante la ejecución de la misma. La medición constante de la reputación online ayuda al hospital a corregir errores y reorientar ciertas iniciativas de comunicación. La reputación de marca se construye a través de un proceso social 
continuo en el que el valor se co-crea a través de la interacción de la compañía con sus stakeholders (Füller, Schroll, Dennhardt y Hutter, 2012). Este proceso da lugar a las comunidades de marca, las cuales influyen positivamente en la confianza y la lealtad que los stakeholders manifiestan hacia la marca (Laroche, Habibi y Richard, 2013).

\section{Conclusión}

Las redes sociales se han convertido en una herramienta capaz de influenciar positivamente en las percepciones que los stakeholders tienen sobre la marca de un hospital. De ahí el interés que el Director de Comunicación de un hospital priorice el uso de las redes sociales para alcanzar los distintos objetivos establecidos en el Plan de Comunicación Institucional. El poder dinamizador de las redes sociales, así como los nuevos roles comunicativos asumidos por los pacientes y los profesionales de la salud, permiten afirmar que la hipótesis planteada al principio de esta investigación es cierta; es decir, la comunicación institucional en las redes sociales se ha convertido en la acción comunicativa más importante para todos aquellos hospitales que desean potenciar su reputación corporativa. Con el objetivo de concluir este artículo, se ofrecen tres últimas ideas que permiten ilustrar el impacto de las redes sociales en la reputación online de una marca hospitalaria. En primer lugar, el Director de Comunicación de un hospital debe contar con un equipo de trabajo multidisciplinar que esté formado por profesionales de la comunicación institucional, pero también por profesionales de la salud, informáticos, diseñadores y matemáticos, ya que solo de este modo podrá lograr que las redes sociales se conviertan en un producto comunicativo capaz de mejorar la reputación online del hospital e influenciar positivamente en la labor diaria de los profesionales de la salud que trabajan en el centro hospitalario. En segundo lugar, el Director de Comunicación debe gestionar las redes sociales de tal modo que consiga crear un patrimonio institucional que sobrepase su mandato como directivo y que ayude al hospital a proyectar su marca en el largo plazo y a reforzar el posicionamiento estratégico del hospital en su mercado de referencia. Y, en tercer lugar, las redes sociales deben ser consideradas como meras herramientas neutras, cuyo impacto positivo en términos de reputación online de marca depende de la implicación de los profesionales de la salud en las distintas iniciativas emprendidas en las redes sociales por parte del Departamento de Comunicación Institucional del hospital. 


\section{Referencias bibliográficas}

Abratt, R., y Kleyn, N. (2012). Corporate identity, corporate branding and corporate reputations. European Journal of Marketing, 46 (7/8), 1048-1063. doi: 10.1108/03090561211230197

Adams, S. (2010). Revisiting the online health information reliability debate in the wake of web 2.0: an interdisciplinary literature and website review. International Journal of Medical Informatics, 79 (6), 391-400. doi: http://dx.doi.org/10.1016/j. ijmedinf.2010.01.006

Alpert, J., y Womble, F. (2016). Just What the Doctor Tweeted: Physicians' Challenges and Rewards of Using Twitter. Health Communication, 31(7), 824-832. doi: http://dx.doi.org/10.1080/10410236.2015.1007551

Andersen, M. (2010). Creating esprit de corps in times of crisis. Corporate Communications: An International Journal, 15(1), 102-123. doi: $10.1108 / 13563281011016868$

Antheunis, M., Tates, K., y Nieboer, T. (2013). Patients' and health professionals' use of social media in health care: Motives, barriers and expectations. Patient Education and Counselling, 92(3), 426-431. doi: http://dx.doi.org/10.1016/j. pec.2013.06.020

Bernhardt, J., Mays, D., y Kreuter, M. (2011). Dissemination 2.0: Closing the Gap Between Knowledge and Practice With New Media and Marketing. Journal of Health Communication, 16:sup1, 32-44, doi: 10.1080/10810730.2011.593608

Becerra, E., Reina, J. y Victoria, J. (2015). Comunicación e imagen de los servicios sanitarios. El caso de los centros hospitalarios andaluces (2004-2013). Prisma Social. Revista de Ciencias Sociales, 14, 0-28.

Britnell, M. (2011). Increasing importance of social media in healthcare. KPMG International, 8, 1-22.

Bubien, Y. (2015). Hôpital 2.0: du virtuel au réel. European Psychiatry, 30(8), S74.

Cady, S., Wheeler, J., DeWolf, J., y Brodke, M. (2011). Mission, vision and values: what do they say? Organizational Development Journal, 29(1), 63-78.

Campbell, B., y Craig, Clay (2014). Social media and health: current and future healthcare provider perspectives. Journal of Contemporary Medical Education, 2(2), 128-133. doi: 10.5455/jeme.20140515123200

Chun, R.(2005).Corporate reputation: meaning and measurement. International Journal of Management Reviews, 7(2),91-109.doi: 10.1111/j.1468-2370.2005.00109.x

Cormode, G., y Krishnamurthy, B. (2008). Key differences between web 1.0 and web 2.0. First Monday, 13(6), 2.

Desmidt, S., Prinzie, A., y Decramer, A. (2011). Looking for the value of mission statements: a meta-analysis of 20 years of research. Management Decision, 49(3), 468-483. doi: 10.1108/00251741111120806

Dijkmans, C., Kerhof, P., y Beukeboom, C. (2014). A stage to engage: Social media use and corporate reputation. Tourism Management, 47, 58-67. doi: https://doi. org/10.1016/j.tourman.2014.09.005

Ditlevsen, M. (2012). Revealing corporate identities in annual reports. Corporate Communications: An International Journal, 17(3), 379-403. doi: $10.1108 / 13563281211253593$. 
Drake, S., Gulman, M., y Roberts, S. (2005). Light their fire: using internal marketing to ignite employee performance and wow your customers. Chicago: Dearborn Trade Publishing.

Fombrun, C., Gardberg, N., y Sever, J. (2000). The reputation quotient: a multiple stakeholder measure of corporate reputation. Journal of Brand Management, 7(4), 241-255.

Fordis, M., Street, R., Volk, R., y Smith, Q. (2011). The Prospects for Web 2.0 Technologies for Engagement, Communication, and Dissemination in the Era of Patient-Centered Outcomes Research: Selected Articles Developed From the Eisenberg Conference Series 2010 Meeting. Journal of Health Communication, 16(1), 3-9. doi: 10.1080/10810730.2011.598398

Füller, J., Schroll, R., Dennhardt, S., y Hutter, K. (2012). Social brand value and the value enhancing role of social media relationships for brands. Proceedings of the $45^{\text {th }}$ Annual Hawaii International Conference of System Sciences ( $p p .3218$ 3227). Maui: Computer Society Press.

Gage-Bouchard, E., LaValley, S., Mollica, M., y Beaupin, L. (2016). Communication and Exchange of Specialized Health-Related Support Among People With Experiential Similarity on Facebook. Health Communication, 2, 1-8. doi: http:// dx.doi.org/10.1080/10410236.2016.1196518

Gao, G., McCullough, J., Agarwal, R., y Jha, A. (2012). A changing landscape of physician quality reporting: analysis of patients' online ratings of their physicians over a 5-year period. Journal of Medical Internet Research, 14, e38. doi: 10.2196/jmir.2003

George, D., Rovniak, L., y Kraschnewski, J. (2013). Dangers and opportunities for social media in medicine. Clinical Obstetrics and Gynecology, 56(3), 453-462. doi: 10.1097/GRF.0b013e318297dc38

Gibbons, C., Fleisher, L., Slamon, R., Bass, S., Kandadai, V., y Beck, R. (2011) Exploring the Potential of Web 2.0 to Address Health Disparities. Journal of Health Communication, 16(1), 77-89. doi: 10.1080/10810730.2011.596916

Greaves, F., Ramirez, D., Millett, C., Darzi, A., y Donaldson, L. (2013). Harnessing the cloud of patient experience: Using social media to detect poor quality healthcare. BMJ Quality \& Safety, 22(3), 251-255. doi: 10.1136/ bmjqs-2012-001527

Griffis, H., Kilaru, A., Werner, R., Asch, D., Hershey, J., Hill, S., Ha, Y., Sellers, A., Mahoney, K., y Merchant, R. (2014). Use of Social Media Across US Hospitals: Descriptive Analysis of Adoption and Utilization. Journal of Medical Internet Research, 16(11), e264. doi: 10.2196/jmir.3758

He, H., y Balmer, J. (2013). A grounded theory of the corporate identity and corporate strategy dynamic. European Journal of Marketing, 47(3-4), 401-430. doi: 10.1108/03090561311297391

He, H., y Balmer, J. (2008). Identity studies: multiple perspectives and implications for corporate-level marketing. European Journal of Marketing, 41(7/8), 765-785. doi: 10.1108/03090560710752393

Hoon Kim, K., Sik Kim, K., Yul Kim, D., Ho Kim, J., y Hou Kang, S. (2008). Brand equity in hospital marketing. Journal of Business Research, 61(1), 75-82. doi: 10.1016/j.jbusres.2006.05.010 
Jaakson, K. (2010). Management by values: are some values better than others? Journal of Management Development, 29(9), 795-806. doi: 10.1108/02621711011072504

Kantabutra, S., y Avery, G. (2010). The power of vision: statements that resonate. Journal of Business Strategy, 31(1), 37-45. doi: 10.1108/02756661011012769

Kemp, E., Jillapalli, R., y Becerra, E. (2014). Healthcare branding: developing emotionally based consumer brand relationships. Journal of Services Marketing, 28(2), 126-137. doi: 10.1108/JSM-08-2012-0157

Kietzmann, J., Hermkens, K., McCarthy, I., y Silvestre, B. (2011). Social media? Get serious! Understanding the functional building blocks of social media. Business Horizons, 54(3), 241-251. doi: http://dx.doi.org/10.1016/j.bushor.2011.01.005

Klein, A. (2011). Corporate culture: its value as a resource for competitive advantage. Journal of Business Strategy, 32(2), 21-28. doi: 10.1108/02756661111109743

Laroche, M., Habibi, M., y Richard, M. (2013). To be or not to be in social media: How brand loyalty is affected by social media? International Journal of Information Management, 33(1), 76-82. doi: 10.1016/j.ijinfomgt.2012.07.003

Lee, H., Lee, C., y Wu, C. (2011). Brand image strategy affects brand equity after M\&A. European Journal of Marketing, 45(7/8), 1091-1111. doi: 10.1108/03090561111137624

Lim, W. (2016). Social media in medical and health care: opportunities and challenges. Marketing Intelligence \& Planning, 34(7), 964 - 976. doi: http://dx.doi. org/10.1108/MIP-06-2015-0120

Marshall, J.,y Adamic, M.(2010). The story is the message: shaping corporate culture. Journal of Business Strategy, 31(2), 18-23. doi: 10.1108/02756661011025035

Matarín Jiménez, T. (2015). Redes sociales en prevención y promoción de la salud. Una revisión de la actualidad. Revista Española de Comunicación de Salud, 6(1), 62-69.

McCarroll, M., Armbruster, S., Chung, J., Kim, J., McKenzie, A., y Von Gruenigen, V. (2014). Health Care and Social Media Platforms in Hospitals. Health Communication, 29(9), 947-952. doi: 10.1080/10410236.2013.813831

Medina Aguerrebere, P. (2015). Management of the internal communication in hospitals: conceptual framework and implementation model. The International Journal of Communication and Health, 5, 53-65.

Metzger, M., y Flanagin, A. (2011). Using Web 2.0 Technologies to Enhance Evidence-Based Medical Information. Journal of Health Communication, 16(1), 45 58. doi: 10.1080/10810730.2011.589881

Naveen, K., Anil, J., y Smruthi, T. (2014). Impact of healthcare marketing and branding on hospital services. International Journal of Research Foundation of Hospital \& Healthcare Administration, 2(1), 19-24. doi: 10.5005/ jp-journals-10035-1010

Nelson, W., Taylor, E., y Walsh, T. (2014). Building an Ethical Organizational Culture. The health care manager, 33(2), 158-164. doi: 10.1097/HCM.0000000000000008

Nieto, A. (2005). Comunicación institucional: bases para la evaluación. En: M. Sevillano (coord.), Reflexiones en torno a la libertad de empresa informativa (pp. 409-442), Madrid: Facultad de Ciencias de la Información. Universidad Complutense de Madrid. 
O’Cass,A.y Weerawardena,J.(2010). The effect of perceived industry competitive intensity and marketing-related capabilities: drivers of superior brand performance. Industrial Marketing Management, 39(4), 571-581.doi: 10.1016/j.indmarman.2009.04.002

Park, H., Rodgers, S., y Stemmle, J. (2013). Analyzing Health Organizations' Use of Twitter for Promoting Health Literacy. Journal of Health Communication, 18(4), 410-425. doi: http://dx.doi.org/10.1080/10810730.2012.727956

Peñafiel Saiz, C., Pastor González, J. M. y Camacho Markina, I. (2014). La información de salud en el universo web dirigida a la juventud: entre profiláctica y educativa. En Carmen Peñafiel; Lázaro Echegaray (Coords.), Estudios de Comunicación y Salud, CAC 63. Tenerife: Sociedad Latina de Comunicación Social. ISBN: 13: 84-15698-65-4.

Pirraglia, P., y Kravitz, R. (2012). Social media: new opportunities, new ethical concerns. Journal of General Internal Medicine, 28(2), 165-166. doi: 10.1007/ s11606-012-2288-x

Rando Cueto, D., Paniagua Rojano, F. y De las Heras Pedrosa, C. (2016). Influence factors on the success of hospital communication via social networks. En: Revista Latina de Comunicación Social, 71. doi: 10.4185/RLCS-2016-1140en

Ratzan, S. (2011). Our New "Social" Communication Age in Health. Journal of Health Communication: International Perspectives, 16(8), 803-804. doi: 10.1080/10810730.2011.610220

Rothberg, M., Morsi, E., Benjamin, E., Pekow, P., y Lindenauer, P. (2008). Choosing the best hospital: the limitations of public quality reporting. Health Affairs, 27(6), 1680-1687. doi: 10.1377/hlthaff.27.6.1680.

Salathé, M., Freifeld, C., Mekaru, S., Tomasulo, A., y Brownstein, J. (2013). Influenza A (H7N9) and the importance of digital epidemiology. The New England Journal of Medicine, 369(5), 401-404. doi: 10.1056/NEJMp1307752

Singal, A., y Jain, A. (2013). An empirical examination of the influence of corporate vision on internationalization. Strategic Change, 22(5-6), 243-257. doi: 10.1002/ jsc. 1937

Smailhodzic, E., Hooijsma, W., Boonstra, A., y Langley, D. (2016). Social media use in healthcare: A systematic review of effects on patients and on their relationship with healthcare professionals. BMC Health Services Research, 16, 442. doi: 10.1186/s12913-016-1691-0

Sparer, M. (2011). US healthcare reform and the future of dentistry. American Journal of Public Health, 10(10), 1841-1844.

Tejedo, F. (2013). Estrategias de comunicación corporativa de las empresas socialmente responsables: análisis del capital relacional como base de las relaciones empresa-stakeholders. ZER Revista Estudios de Comunicación, 35, 191-213.

Trong, L. (2014). Corporate governance and brand performance. Management Research Review, 37(1), 45-68. doi: 10.1108/MRR-08-2012-0183

Vance, K., Howe, W, y Dellavale, R. (2009): Social internet sites as a source of public health information. Dermatologic Clinic, 27(2), 133-136. doi: 10.1016/j. det.2008.11.010

Van Riel, C. (1998). Comunicación corporativa (Trad. E. Cerdá). Madrid: Prentice Hall. Van Riel, C., y Fombrun, C. (2007). Essentials of corporate communication: implementing practices for effective reputation gestion. Abingdon: Routledge. 
Visa Barbosa, M., y Coll-Planas, G. (2015). Compartir la enfermedad online: narrativas de restitución y búsqueda en blogs de mujeres con cáncer de mama. ZER Revista de Estudios de Comunicación, 35, 195-210.

Whiteley, A., Price, C., y Palmer, R. (2013). Corporate culture change: adaptive culture structuration and negotiated practice. Journal of Workplace Learning, 25(7), 476-498. doi: 10.1108/JWL-09-2012-0069

Würz, A., Nurm, Ü., y Ekdahl, K. (2013). Enhancing the Role of Health Communication in the Prevention of Infectious Diseases. Journal of Health Communication, 18(12), 1566-1571. doi: 10.1080/10810730.2013.840698 